\title{
The Effects of a Gentle Yoga Program on Sleep, Mood, and Blood Pressure in Older Women with Restless Legs Syndrome (RLS): A Preliminary Randomized Controlled Trial
}

\author{
Kim E. Innes ${ }^{1,2}$ and Terry Kit Selfe ${ }^{1,2}$ \\ ${ }^{1}$ Department of Community Medicine, West Virginia University School of Medicine, P.O. Box 9190, Morgantown, WV 26506-9190, \\ USA \\ ${ }^{2}$ Center for the Study of Complementary and Alternative Therapies, University of Virginia Health System, P.O. Box 800782, \\ McLeod Hall, Charlottesville, VA 22908-0782, USA
}

Correspondence should be addressed to Kim E. Innes, kinnes@hsc.wvu.edu

Received 21 November 2011; Accepted 21 December 2011

Academic Editor: David Mischoulon

Copyright ( $\odot 2012$ K. E. Innes and T. K. Selfe. This is an open access article distributed under the Creative Commons Attribution License, which permits unrestricted use, distribution, and reproduction in any medium, provided the original work is properly cited.

\begin{abstract}
Objective. To examine the effects of yoga versus an educational film program on sleep, mood, perceived stress, and sympathetic activation in older women with RLS. Methods. Participants were drawn from a larger trial regarding the effects of yoga on cardiovascular disease risk profiles in overweight, sedentary postmenopausal women. Seventy-five women were randomized to receive either an 8 -week yoga $(n=38)$ or educational film $(n=37)$ program. All 75 participants completed an RLS screening questionnaire. The 20 women who met all four diagnostic criteria for RLS ( $n=10$ yoga, 10 film group) comprised the population for this nested study. Main outcomes assessed pre- and post-treatment included: sleep (Pittsburgh Sleep Quality Index), stress (Perceived Stress Scale), mood (Profile of Mood States, State-Trait Anxiety Inventory), blood pressure, and heart rate. Results. The yoga group demonstrated significantly greater improvements than controls in multiple domains of sleep quality and mood, and significantly greater reductions in insomnia prevalence, anxiety, perceived stress, and blood pressure (all $P$ 's $\leq 0.05$ ). Adjusted intergroup effect sizes for psychosocial variables were large, ranging from 1.9 for state anxiety to 2.6 for sleep quality. Conclusions. These preliminary findings suggest yoga may offer an effective intervention for improving sleep, mood, perceived stress, and blood pressure in older women with RLS.
\end{abstract}

\section{Introduction}

Restless legs syndrome (RLS) is a distressing and potentially debilitating sleep disorder, affecting up to $29 \%$ of U.S. and European general adult populations, and an estimated average of $19.5 \%$ of primary care patients [1]. RLS is characterized by a compelling urge to move the legs, usually accompanied by uncomfortable sensations in the legs, that: begins or worsens during periods of inactivity, is worse during the evening and nighttime hours, and is partially or totally relieved by movement [2]. Prevalence increases with age, and RLS is approximately twice as common in women as in men [1]. RLS has been repeatedly associated with significant reductions in quality of life comparable to or worse than those reported in Parkinson's disease, diabetes, stroke, and other serious chronic disorders [3-7]. RLS has also been linked to substantial impairment in sleep, mood, and health and is associated with significant societal and economic burden $[4,5,7]$.

While RLS is considered a neurological disorder, the etiology of RLS remains poorly understood [8-10]. Defects in dopamine function and iron metabolism have been considered key factors in the pathogenesis of RLS for decades [11-14], based largely on clinical observations [14-18], although evidence remains inconclusive $[8,15,19,20]$. An emerging body of evidence supports a potential role for autonomic and metabolic dysfunction. Recent studies have linked RLS to cardiovascular disease (CVD), as well as to key components of the metabolic syndrome, including diabetes, obesity, hypertension, and dyslipidemia, conditions 
associated with both autonomic and metabolic dysregulation [21]. RLS is characterized by elevated nocturnal blood pressure and heart rate [22-24], increased nocturnal hypothalamic-pituitary-adrenal (HPA) axis activation [25], elevated sympathetic activation, and reduced parasympathetic tone [26, 27], suggesting that autonomic and HPA axis dysfunction may in part underlie the development and/or progression of RLS and could help explain the observed association of RLS to CVD and related disorders [21].

There is no cure for RLS. Existing treatments are aimed at symptom reduction and include dopaminergic agents, opioids, sedative hypnotics, anticonvulsants, and benzodiazepines. Unfortunately, these medications can have serious side effects. For example, common side effects of dopaminergic agents, considered the first-line pharmacologic treatment for RLS sufferers $[4,16,28]$, include rebound and augmentation of symptoms, dyskinesia, nausea and vomiting, hypotension, impulse control disorders, dizziness, and insomnia or drowsiness [7, 29-31]; common side effects of other RLS medications include potential for dependence, confusion, blurred vision, coordination problems, and other adverse sequellae $[7,29,30]$. These effects can be particularly problematic in older populations [32, 33], who also suffer disproportionately from RLS [29, 34]. In addition, the benefits of these medications may diminish over time [35, 36], leaving patients with few treatment options.

Given the substantial drawbacks of these pharmacologic treatments, investigation of safe, nonpharmacologic therapies that address apparent underlying risk factors is needed. Yet research remains very sparse. For example, there have to date been only three small trials examining the potential benefits of lifestyle or behavioral interventions for individuals suffering from RLS. These include two studies evaluating the effects of a 12-16 week exercise program versus usual care on RLS symptoms [37, 38], and a recent pre-post trial assessing the effects of cognitive behavioral therapy (CBT) in adults with primary RLS and mild-moderate psychosocial impairment [39]. Investigators reported significant improvements in RLS-related outcomes relative to the control group [37, 38] or baseline [39], suggesting that nonpharmacologic interventions may benefit those suffering from RLS.

Yoga, an ancient mind-body discipline that is increasingly popular in the U.S. [40], may represent a particularly promising nonpharmacologic therapy for RLS. Accumulating evidence from controlled trials suggests yoga can reduce blood pressure [41], improve glucose tolerance [41-43], lipid profiles $[41,44,45]$, body composition $[41,45]$, and autonomic function [42, 44, 45], enhance mood [46-49], and improve sleep $[48,50,51]$, factors linked to both RLS and CVD risk $[8,9,16,21]$. In addition, studies suggest yoga can decrease muscular excitability and induce more rapid muscle relaxation [52], factors that have also been implicated in RLS $[53,54]$. However, while yoga and other relaxation therapies are often recommended for RLS patients [55, 56], clinical trials are lacking. In this small, nested RCT, we investigated the effects of yoga on sleep, mood, stress, and associated outcomes in older women with RLS.

\section{Methods}

Participants for this study were drawn from a feasibility study regarding the effects of yoga on CVD risk profiles in sedentary, overweight postmenopausal women [57]. In this larger RCT, 75 women aged 45-79 years were recruited from the community using a combination of flyers, brochures, and newspaper advertisements. Eligible participants were nonsmoking women at least 45 years of age who were postmenopausal ( $\geq 12$ months amenorrheic), physically inactive (exercising less than 20 minutes, 3 times per week), and overweight (body mass index (BMI, calculated as $\mathrm{kg} / \mathrm{m}^{2}$ ) $\geq 25$, and/or waist circumference $\geq 88 \mathrm{~cm}$ ) or had a first-degree relative with diabetes or essential hypertension. Excluded were women who had practiced yoga within the last year, had uncontrolled hypertension, had been diagnosed with sleep apnea or with diabetes, cancer, heart failure, or other serious chronic disorders, or had any orthopedic, neurological, or other condition that might prevent them from safely completing an 8-week yoga program. Following enrollment, screening, and baseline assessment, eligible participants were randomized, using a computer-generated randomization list provided by a statistician not associated with the study, to receive either a gentle 8 -week yoga program $(n=38)$ or an 8 -week educational film program $(n=37)$. Each participant was administered her treatment assignment sequentially in order of enrollment, via coded opaque envelopes containing general study instructional materials, a welcome letter, and information pertinent to the yoga or educational film program. The study was approved by the University of Virginia Health Sciences Research Institutional Review Board, and all participants provided informed consent prior to study enrollment.

2.1. RLS and Outcome Assessment. Participants completed an RLS screening questionnaire at baseline. The questionnaire was adapted by the authors from the Athens Sleep Center Screening Questionnaire for RLS (http://www.athenssleepcenter.com/PDF/AScreeningQuestionaireRLS.pdf) to incorporate the established four criteria for RLS established by the International Restless Legs Syndrome Study Group (IRLSSG) [2]. Participants were asked if they ever experienced an urge to move with uncomfortable/funny (e.g., tingling, creeping, crawling) sensations in the legs, and if these feelings began or worsened when lying or sitting, were worse in the evening/night, and were relieved at least in part by movement (e.g., wiggling feet, toes, or walking). The questionnaire also included an item regarding frequency of symptoms (never, occasionally $(<1 \mathrm{x} /$ month), sometimes ( $1-$ $3 x /$ month), frequently (1-2x/week to daily), or only in the past). Those participants endorsing all 4 diagnostic criteria and reporting symptoms at least once per month $(N=20)$ were considered to meet diagnostic criteria for RLS and were included in the present study.

Participants underwent a comprehensive assessment at baseline and following the 8-week intervention period. All assessments were conducted by experienced General Clinical Research Center and university clinical laboratory staff blinded to participant treatment assignment. Detailed 
baseline information was gathered on medical history, demographic characteristics, and lifestyle factors. Primary outcomes for this substudy included well-validated selfreport measures of sleep (Pittsburgh Sleep Quality Index (PSQI)) [58], stress (Perceived Stress Scale (PSS)) [59], mood (Profile of Mood States (POMS)) [60], and anxiety (StateTrait Anxiety Inventory (STAI)) [61], as well as indices of sympathetic activation (blood pressure, heart rate). Clinical insomnia was defined as a score of greater than 5 on the PSQI $[58,62]$. Blood pressure and heart rate were measured in a supine position following a 5-minute rest period; measurements were taken 3 times and averaged for a final score.

Additional outcomes included indices of abdominal adiposity (waist circumference $(\mathrm{cm})$ and BMI $\left(\mathrm{kg} / \mathrm{m}^{2}\right)$ ). At baseline, all women were screened for prediabetes (fasting glucose $100-125 \mathrm{mg} / \mathrm{dL}$ )/diabetes (fasting glucose $\geq 126 \mathrm{mg} / \mathrm{dL}$ ), and serum levels of ferritin were measured. Blood samples were collected in the morning following an overnight fast and drawn by a trained phlebotomist using Vacutainer tubes. Blood samples (ferritin) were stored at $-70^{\circ} \mathrm{C}$ until assay. Glucose was assessed using a Beckman glucose analyzer. Ferritin levels were measured using the ARCHITECT ferritin assay, a Chemiluminescent Microparticle Immunoassay (CMIA) for the quantitative determination of ferritin in human serum and plasma.

To assess the possible influence of physical activity and social support on change in outcomes over time, factors implicated in RLS [63] and strongly linked to both sleep and mood [64], participants completed the Physical Activity Scale for the Elderly (PASE) [65] and the Duke Social Support Index (abbreviated form [66]) pre- and posttreatment. To measure expectations of benefit, a brief treatment expectancy questionnaire was also administered to all participants. In addition, all subjects were asked to complete a short, anonymous exit questionnaire regarding their experience with the study.

2.2. Intervention. Each group (yoga and film intervention) attended a 90-minute class twice weekly for 8 weeks. Classes for the two programs were scheduled at the same times and in similar settings. Class size in both groups was limited to no more than 14 participants. Upon completing the study, all participants were given $\$ 150$ for their time and travel expenses. Film group participants also received the yoga home practice materials, as well as coupons to attend local Iyengar yoga classes. Similarly, yoga group participants were also offered the opportunity to view the educational films following completion of the final assessment.

2.2.1. Yoga Intervention. Participants randomized to the yoga program completed a gentle Iyengar yoga program specifically designed for older, sedentary adults. In addition to attending classes, participants were asked to complete at least 30 minutes of home practice on nonclass days, with the aid of a DVD and a booklet illustrating the yoga home practice routines. Designed and taught by a senior Iyengar yoga instructor with over 30 years of experience, the yoga program included the following 23 active and restorative poses (asanas): Mountain (Tadasana and Urdhva hastasana in Tadasana); Standing wide apart legs (Prasarita padottanasana); Warrior I and II (Virabhadrasana I and II); Fierce or chair pose (Utkatasana); Extended hands and feet pose (Utthita hasta padasana); 1/2 forward bend (Ardha uttanasana); Triangle (Utthita trikonasana); Reverse triangle (Parivrtta trikonasana); Downward facing dog (Adho mukha svanasana); Marichi's pose (Marichyasana I and III, Utthita marichyasana); Seated twist in chair(Bharadvajasana); One leg straight forward spinal stretch (Janu sirsasana); Seated wide angle (Upavista konasana and Parsva upavista konasana); Seated bound angle (Baddha konasana); Extended legs up the wall (Urdhva prasarita padasana); Lying down holding big toe (Supta padangusthasana); Turned around belly (Jathara parivartanasana); Supported boat (Navasana); Supported bridge (Setu bandha sarvangasana); Crossed legs back arch (Supta swastikasana); Lying down bound angle (Supta baddha konasana); Reclined mountain (Supta tadasana); and Corpse pose (Savasana). Poses were modified and props (blankets, chairs, and straps) used as needed to allow participants to perform the sequences easily and safely. Each session began with a simple yogic centering and breathing exercise and ended with a 10-15 minute guided supine relaxation practice (Savasana).

2.2.2. Educational Film (Control) Intervention. This standardized educational film program, described in detail elsewhere [57], was designed to be easily replicable and to ensure comparability of staff attention, class time, and social interaction. Each class began with a brief meet and greet period, followed by viewing of an educational film chosen for its relevance and interest to our study population, then concluded with a 10-15 minute group discussion facilitated by a health professional with expertise in complementary and alternative therapies and women's health. To increase credibility and reduce participant bias, the educational film program was presented to potential participants as an informative, engaging, and relaxing alternative to the yoga classes.

2.3. Adherence and Adverse Events. Attendance at both the yoga and the film classes was recorded by the respective instructors. Participants in the yoga class also completed a check sheet and log after each home practice session, indicating the number of minutes practiced and any comments they might have regarding the session. Homework logs were turned in to the instructor at the first group class each week.

Adverse events were tracked via weekly review of participant yoga logs. At the beginning of each class, the yoga instructor also queried participants regarding potential problems; these were likewise recorded. In addition, participants were encouraged to contact study investigators and/or staff regarding any potential concerns.

2.4. Statistical Analysis. Data were analyzed using PASW v. 18. Differences between those who did versus did not meet criteria for RLS at baseline, and between yoga and 
control group participants at baseline were evaluated using chi-square (for categorical variables), student independent samples $t$ tests (for continuous variables with a normal distribution), or Mann-Whitney $U$ tests (for ordinal variables or continuous variables with evidence of skewing). Within group changes over time (pre- to postintervention) were evaluated using chi-square or McNemar test (categorical variables), paired t tests (continuous variables), or Wilcoxon signed rank test (ordinal or continuous variables with evidence of skewing). Between-group differences over time were assessed using Repeated Measures ANOVA (multivariate tests). Distributions of all dependent variables were examined to ensure the assumptions of normality and sphericity were met and variables transformed as necessary. Between-group differences over time in categorical variables were assessed using logistic regression. Effect sizes were calculated using Cohen's $d$ [67], with between-group effect size adjusted for differences in baseline values.

\section{Results}

Twenty (27\%) of the 75 women enrolled in the parent trial met the IRLSSG four essential diagnostic criteria for RLS, with symptoms at least once per month $(n=10$ yoga group, 10 film group participants). Only 2 (10\%) of the 20 reported having received an RLS diagnosis; neither were taking RLS medications. Of those with RLS, 17 ( 8 yoga, 9 control) experienced symptoms at least once/week. Baseline characteristics of the participants with versus without RLS are given in Table 1. Relative to those without RLS, women with RLS demonstrated significantly poorer sleep quality, greater prevalence of insomnia (85\% versus 38\% for those with versus without RLS, $P=0.0002)$, shorter sleep duration $(6.2 \pm 0.3$ versus $7.1 \pm 0.2$ hours, $P=0.002)$, and higher diastolic and systolic blood pressure $(P<0.005)$ and were more likely to report a history of depression $(P<0.01)$. Participants with RLS were less likely to specify a history of hormone replacement therapy $(P=0.03)$ and indicated a significantly higher number of pregnancies than those without RLS $(X=2.9 \pm 0.5$ versus $1.8 \pm 0.2$, resp., $P=$ 0.02). Those with RLS also tended to report higher trait anxiety, greater mood impairment, and lower vigor $(P<$ $0.1)$. Women with RLS did not differ from those without RLS in baseline ferritin levels $(81 \pm 10.5$ versus $84.7 \pm 8.4 \mathrm{ng} / \mathrm{mL}$, resp., $P=0.8)$ or in the prevalence of low ferritin levels $(<$ $50 \mathrm{ng} / \mathrm{mL}$, $30 \%$ versus $36 \%$, resp., $P=0.6$ ).

Of those 20 participants screening positive for RLS, 5 (25\%) were African American, 65\% had completed at least 4 years of college, $65 \%$ were employed at least part time, $35 \%$ were married, and $50 \%$ were pre-diabetic (fasting glucose $100-125 \mathrm{mg} / \mathrm{dL})$. Participant age averaged $58.8 \pm 0.9$ years. As indicated in Table 2, treatment and control groups did not differ in demographic characteristics, or in baseline lifestyle factors, anthropometric characteristics, medical history, health profiles, prevalence of insomnia, or reported frequency of RLS symptoms. Mood and sleep profiles were also similar between the groups $(P>0.3$, data not shown). Eighteen of the 20 participants with RLS completed the final assessment (8 yoga, 10 controls). Those leaving the study (one non-Hispanic white, one African American) dropped out during the first 2-3 weeks due to health reasons unrelated to the yoga program. Adherence in both the yoga and the educational film group was very good overall. Class attendance in the two groups was similar (mean attendance $=13.1 \pm 1.4$ versus $13.6 \pm 0.8$ classes (of 16 total classes) for the yoga versus film group participants, respectively, $P=0.75)$. Yoga group participants completed homework practice a mean of $4.1 \pm 0.2$ days/week, with an average $28.0 \pm$ 3.3 minutes/practice session. Participant feedback on openended exit questionnaires was also positive, with participants of both groups expressing enthusiasm for their respective programs [57]. Of the 8 yoga group participants completing the study, all cited high satisfaction with the yoga program and all reported multiple benefits on their yoga logs and/or exit questionnaires, including increased strength, flexibility and mobility $(N=6)$, reduced pain $(N=3)$, enhanced energy and well-being $(N=6)$, increased feelings of peace, tranquility, and relaxation $(N=8)$, and greater body awareness $(N=4)$. While three yoga group participants indicated some mild, temporary muscle soreness in the first few weeks, no participants reported significant pain, discomfort or other adverse events in association with the yoga program.

As illustrated in Table 3, yoga group participants showed significant reduction over time in prevalence of insomnia $(P=0.01)$ and significant improvement in sleep quality, both overall $(P=0.001)$ and in the domains of sleep duration $(P=0.02)$, efficiency $(P=0.01)$, disturbance $(P=0.03)$, and daytime dysfunction $(P=0.002)$. Reported average sleep duration increased from $5.7 \pm 0.5$ to $7.3 \pm 0.5$ hours $(P=0.001)$. Yoga participants also demonstrated significant, or marginally significant improvements in all but one domain of mood $(P \leq 0.08)$, as well as significant reductions in state anxiety, perceived stress, and both systolic and diastolic blood pressure $(P<0.05)$.

Despite limited study power, the yoga group demonstrated significantly greater improvement than controls in several domains of sleep quality, greater reductions in prevalence of insomnia, and greater increases in average sleep duration. Relative to controls, yoga group participants also showed significantly greater reductions in perceived stress, mood disturbance, state anxiety, and both systolic and diastolic blood pressure (all P's $\leq$ 0.05). Adjusted intergroup effect sizes for psychosocial variables were large, with those for summary scores ranging from 1.9 for state anxiety to 2.6 for sleep quality (Table 3); intergroup effect sizes for blood pressure were also substantial, calculated as 0.9 for diastolic blood pressure and 1.25 for systolic blood pressure. Intent to treat analysis, using the conservative last value carried forward method to address missing data, did not appreciably alter these findings. Neither treatment expectancy scores nor change in physical activity or social support differed significantly between the two groups; moreover, adjustment for these variables did not materially alter the results, suggesting that these factors did not explain the observed between-group differences. 


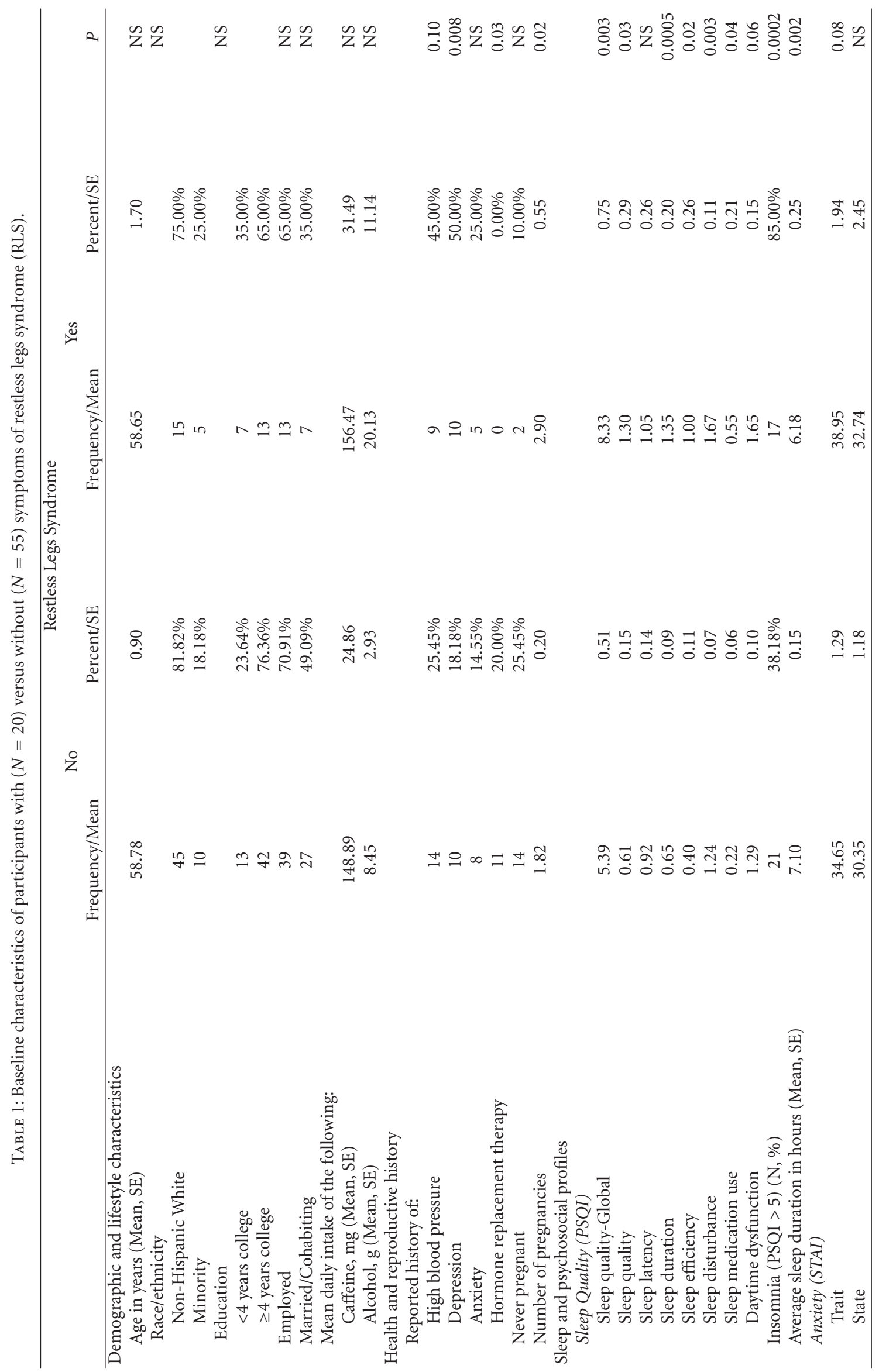




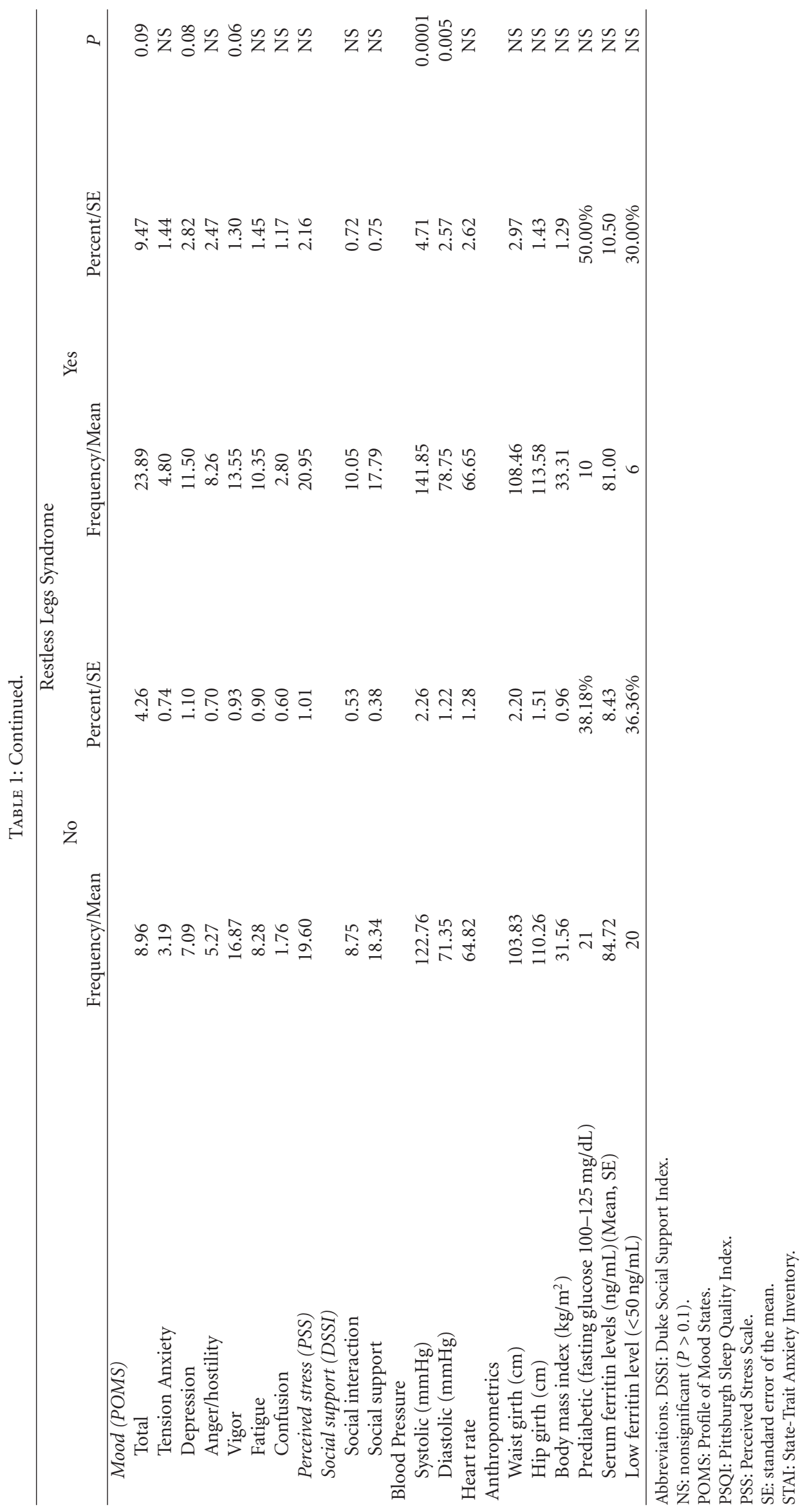


TABLE 2: Baseline characteristics of participants with restless legs syndrome (RLS) assigned to the yoga $(N=10)$ versus the educational film (control) group $(N=10)$.



Abbreviations. NS: nonsignificant $(P>0.5)$.

\section{Discussion}

RLS is a common sleep and sensorimotor disorder associated with significant reductions in quality of life that are largely attributable to the substantial impairment in sleep and mood commonly accompanying RLS [4]. In light of the potentially serious side effects of existing pharmacological treatments for RLS, investigation of promising lifestyle and behavioral interventions is clearly warranted. To our knowledge, this is the first study to examine the effects of yoga in persons with RLS, and among the few trials to examine the potential benefits of any nonpharmacologic intervention for those suffering from this disorder. Findings of this preliminary RCT suggest that yoga can significantly improve sleep, enhance mood, reduce stress and anxiety, and decrease blood pressure in postmenopausal women with RLS, and thus may offer a promising new treatment modality for this population. Moreover, the overall excellent compliance, high participant satisfaction, and lack of adverse events observed in this study suggest that a gentle yoga program is both feasible and acceptable to older women with RLS.

Consistent with our findings, a nonrandomized controlled trial in 14 dialysis patients with RLS reported significant improvements in sleep and mood in those completing a 16-week aerobic exercise program, with adjusted intergroup effect sizes ranging from 0.75 to 1.00 [37]. Likewise, a prepost study of 25 outpatients with psychosocial impairment due to RLS reported significant, although more modest improvements in sleep and mood following 8 weeks of CBT (effect sizes 0.1-0.8) [39], improvements that appeared attributable at least in part to mindfulness, breathing, and other stress-reduction exercises. Although no published studies have assessed the effects of yoga on blood pressure in RLS patients, a number of controlled studies have reported yoga interventions to reduce blood pressure in other both healthy and chronically ill populations [41, 49, 68-70].

While we did not assess the effects of yoga specifically on symptoms of RLS, change in sleep and mood are endpoints recommended for use in clinical trials by the IRLSSG [7] and have been used in trials regarding the efficacy of dopamine agonists and other pharmaceutical treatments for RLS [7176]. Of particular note, the improvements in sleep quality and mood observed following our 8-week yoga program appear comparable to those reported in recent pharmaceutical trials that used similar measures in RLS patients [71, $72,74,76,77]$, suggesting that yoga could possibly provide a viable alternative to pharmaceutical therapy for some patients. Given these promising preliminary findings, larger 


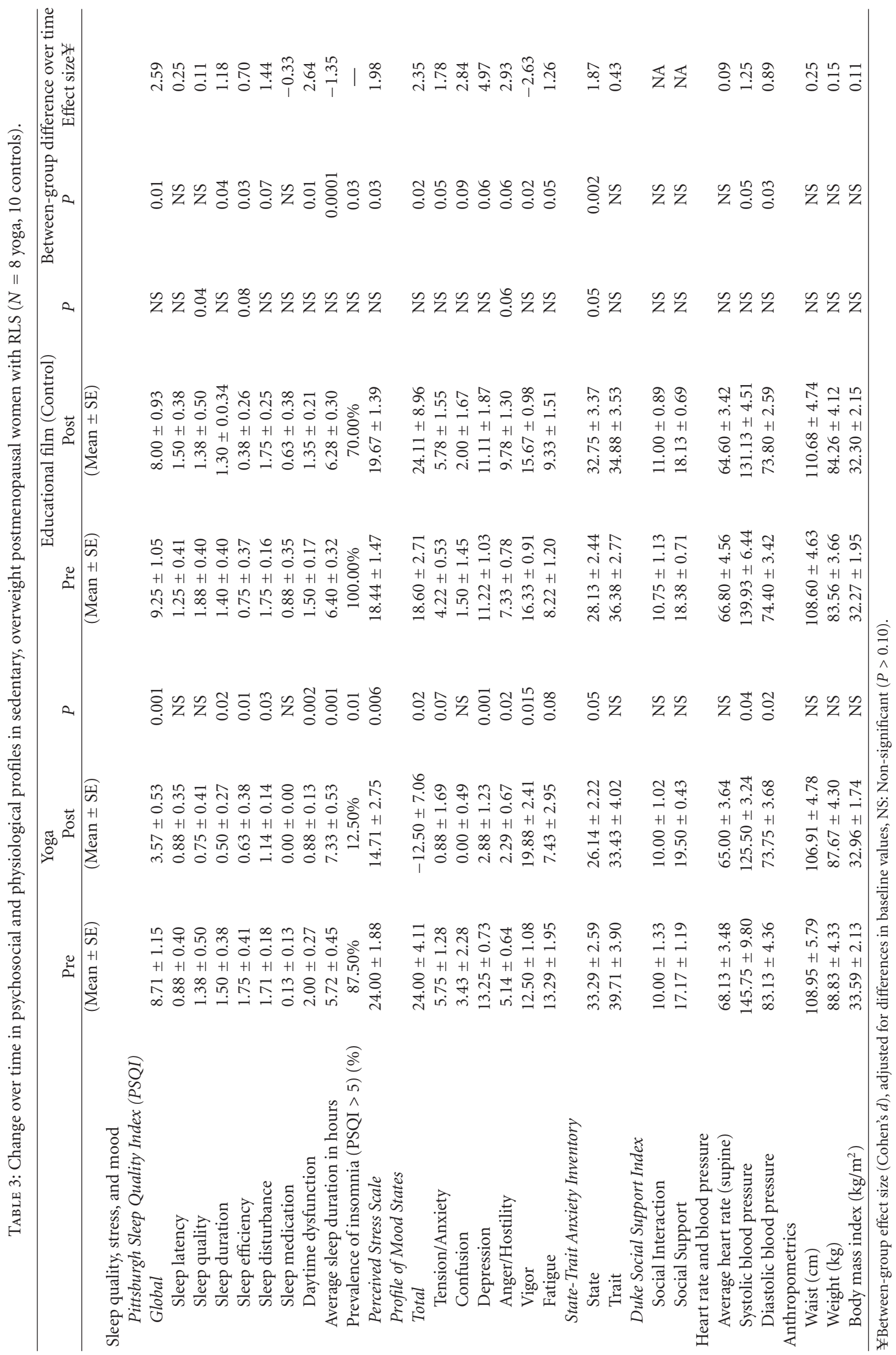






FIGURE 1: Possible pathways by which yoga may attenuate the distressing symptoms associated with RLS.

controlled trials are warranted to investigate the potential utility of yoga as an adjunct or primary treatment for RLS.

Only $10 \%$ of study participants screening positive for RLS had received a physician diagnosis, comparable to the low diagnostic rates generally reported in other, larger studies [78-80]. Consistent with previous research [81-83], participants with RLS reported higher parity and demonstrated significantly greater sleep deficits and mood disturbance than those without RLS, with $85 \%$ indicating insomnia at baseline and 50\% reporting a history of depression. In agreement with several, but not all recent studies [21], RLS was associated with significantly higher blood pressure in this study. Participants with RLS were also less likely to report use of hormone replacement therapy (HRT) than those without RLS. Evidence from recent experimental studies in postmenopausal women suggest HRT may decrease complaints of restless legs [84], improve subjective sleep quality $[85,86]$, suppress muscle sympathetic nerve activity [87], and reduce nocturnal arousals [84], findings consistent with a possible protective effect of HRT for RLS. However, the relation between HRT and RLS remains unclear, with 2 epidemiological studies indicating positive, although nonsignificant, associations between RLS and HRT $[88,89]$, and a French study of 440 postmenopausal women with RLS reporting no relation of HRT use to symptom severity [90].

Although mechanisms underlying the improvements with yoga observed in this study remain speculative, yoga may benefit those with RLS via several possible interrelated pathways, illustrated in Figure 1. For example, by reducing the activation and reactivity of the sympathetic nervous system and the HPA axis, factors recently implicated in RLS etiology $[9,21,25]$ and known to have strong, bidirectional relationships with sleep and mood [91, 92], yoga may attenuate RLS-associated pain and discomfort, reduce perceived stress and promote feelings of well-being, enhance sleep, lower muscle excitability, and foster positive downstream changes in metabolic status, neuroendocrine function, and inflammatory responses. Second, as illustrated in pathway 2, yoga may also alleviate the distressing symptoms of RLS by directly enhancing parasympathetic output, possibly via stimulation of the vagus nerve [93-95], and in this way, shift the autonomic nervous system balance from primarily sympathetic to parasympathetic $[93,95]$. This, in turn, may enhance sleep and mood, reduce perceived pain, promote muscle relaxation, and lead to positive changes in cardiovagal function and related neuroendocrine, metabolic, and inflammatory responses.

Third, findings of recent neuroimaging and neurophysiological studies [93, 96, 97] suggest that yogic practices may, by selectively activating specific neurochemical systems implicated in RLS, likewise promote beneficial changes in sympathetic/parasympathetic balance, in neuroendocrine function, in affect, sleep, and pain processing, and in related metabolic and inflammatory responses (pathway 3). For example, yogic practices have been shown to increase brain levels of dopamine [98], a neurotransmitter long implicated in the development of RLS and thought to play a key role in pain processing [99], sleep [100], motor control [101], and metabolic regulation $[102,103]$. Yoga also increases GABA [97], an inhibitory neurotransmitter involved in the regulation of muscular excitability [104], mood [105], sleep [100], and pain processing [106]; the GABA agonist gabapentin, an anticonvulsant used in treating RLS [73, 77], as well as chronic pain, mood disorders, and insomnia [107$109]$, is thought to operate at least in part by increasing brain GABA $[73,77]$.

4.1. Strengths and Limitations. Strengths of this study include the community-based design, randomization of participants, between-group similarity in baseline characteristics, high participant retention and adherence, and a comparison condition designed to control for time, attention, and social interaction. Limitations of this pilot study are several. Sample 
sizes were small, reducing our power to detect betweengroup differences over time. However, despite limited power, we observed significant improvement in the yoga versus control group in several clinically important parameters, again arguing for a potentially powerful beneficial effect of the yoga program. Although the educational film program was designed to control for staff attention, setting, and class time, control group participants did not receive homework assignments. In addition, while assessors were blinded to participant treatment status, participants could not be masked. However, response to the educational film (control) program was enthusiastic, and both retention and compliance were excellent [57]. Treatment expectancies did not differ between-groups, and adjustment for treatment expectancies did not materially alter findings, suggesting that placebo effects did not explain the observed findings. Moreover, observed effect sizes were substantially larger than would be expected with placebo [110], and significantly greater than those observed in the controls.

The study population was restricted to sedentary, overweight, postmenopausal women, and findings may therefore not be generalizable to other populations. RLS diagnosis was based on self-report, and some degree of diagnostic error is thus likely. However, affirmative response to all 4 essential criteria renders inclusion of mimics less likely [111]. Finally, the parent study was not designed specifically to address effects of yoga practice on RLS, and we did not measure change in RLS symptoms per se. However, as indicated above, we did screen for RLS at baseline and assess change in sleep quality and mood, which are outcomes that have been recommended for inclusion in clinical trials of RLS [7] and often used as secondary endpoints in pharmaceutical trials of RLS patients [112-116]. Significant sleep and mood impairment have been repeatedly documented in studies of RLS $[7,117,118]$. Sleep loss is the most common presenting complaint of patients seeking medical care for RLS [118, 119] and is thought to explain, in large part, the negative effects of RLS on health and quality of life [80].

\section{Conclusions}

These preliminary findings suggest that yoga may offer a safe, beneficial intervention for reducing sleep and mood disturbance, perceived stress, anxiety, and blood pressure in older women with RLS. Larger controlled trials are needed to confirm these benefits in this and other adult populations with RLS, examine the effects of yoga practice specifically on RLS symptoms and symptom severity, and to evaluate potential underlying mechanisms.

\section{Conflict of Interests}

No competing interests or conflict of interest are declared by any of the author.

\section{Acknowledgments}

The authors would like to thank Dr. Ivora Hinton, Dr. Gina Alexander, and Research Assistants Sarah Cottingham,
Sarah Hasan, and Sundas Idrees for their assistance with data collection. They are also grateful to Dr. Ann Taylor for essential logistical support, to Jewel Holmberg for technical assistance, and to Iyengar Yoga Master Sandra Pleasants, who was largely responsible for the design and implementation of the yoga program. This work was performed at the University of Virginia and West Virginia University and was supported by the National Center for Complementary and Alternative Medicine and the Office of Research on Women's Health (Grant nos. R21-AT002982 and 1-K01-AT004108 to K. E. Innes); and the National Center for Research Resources (Grant no. M01-RR00847). The contents are solely the responsibility of the authors and do not represent the official views of the University of Virginia, West Virginia University, or the National Institutes of Health.

\section{References}

[1] K. E. Innes, T. K. Selfe, and P. Agarwal, "Prevalence of restless legs syndrome in North American and Western European populations: a systematic review," Sleep Medicine, vol. 12, no. 7, pp. 623-634, 2011.

[2] R. P. Allen, D. Picchietti, W. A. Hening et al., "Restless legs syndrome: diagnostic criteria, special considerations, and epidemiology. A report from the restless legs syndrome diagnosis and epidemiology workshop at the National Institutes of Health," Sleep Medicine, vol. 4, no. 2, pp. 101-119, 2003.

[3] L. Abetz, R. Allen, A. Follet et al., "Evaluating the quality of life of patients with restless legs syndrome," Clinical Therapeutics, vol. 26, no. 6, pp. 925-935, 2004.

[4] J. P. Reese, K. Stiasny-Kolster, W. H. Oertel, and R. C. Dodel, "Health-related quality of life and economic burden in patients with restless legs syndrome," Expert Review of Pharmacoeconomics and Outcomes Research, vol. 7, no. 5, pp. 503521, 2007.

[5] T. Reinhold, F. Mller-Riemenschneider, S. N. Willich, and B. Brggenjrgen, "Economic and human costs of restless legs syndrome," PharmacoEconomics, vol. 27, no. 4, pp. 267-279, 2009.

[6] S. Happe, J. P. Reese, K. Stiasny-Kolster et al., "Assessing health-related quality of life in patients with restless legs syndrome," Sleep Medicine, vol. 10, no. 3, pp. 295-305, 2009.

[7] C. J. Earley and M. H. Silber, "Restless legs syndrome: understanding its consequences and the need for better treatment," Sleep Medicine, vol. 11, no. 9, pp. 807-815, 2010.

[8] J. W. Winkelman, "Considering the causes of RLS," European Journal of Neurology, vol. 13, no. 2, pp. 8-14, 2006.

[9] A. S. Walters and D. B. Rye, "Review of the relationship of restless legs syndrome and periodic limb movements in sleep to hypertension, heart disease, and stroke," Sleep, vol. 32, no. 5, pp. 589-597, 2009.

[10] J. E. Smith and J. M. Tolson, "Recognition, diagnosis, and treatment of restless legs syndrome," Journal of the American Academy of Nurse Practitioners, vol. 20, no. 8, pp. 396-401, 2008.

[11] W. B. Matthews, "Letter: iron deficiency and restless legs," British Medical Journal, vol. 1, no. 6014, p. 898, 1976.

[12] R. Sandyk, "The restless legs syndrome (Ekbom's syndrome)," South African Medical Journal, vol. 63, no. 18, pp. 701-702, 1983.

[13] S. T. O'Keeffe, "Restless legs syndrome: a review," Archives of Internal Medicine, vol. 156, no. 3, pp. 243-248, 1996. 
[14] R. Allen, "Dopamine and iron in the pathophysiology of restless legs syndrome (RLS)," Sleep Medicine, vol. 5, no. 4, pp. 385-391, 2004.

[15] L. M. Trotti, S. Bhadriraju, and D. B. Rye, "An update on the pathophysiology and genetics of restless legs syndrome," Current Neurology and Neuroscience Reports, vol. 8, no. 4, pp. 281-287, 2008.

[16] K. Ekbom and J. Ulfberg, "Restless legs syndrome," Journal of Internal Medicine, vol. 266, no. 5, pp. 419-431, 2009.

[17] C. Trenkwalder, B. Högl, and J. Winkelmann, "Recent advances in the diagnosis, genetics and treatment of restless legs syndrome," Journal of Neurology, vol. 256, no. 4, pp. 539 $553,2009$.

[18] C. Trenkwalder and W. Paulus, "Restless legs syndrome: pathophysiology, clinical presentation and management," Nature Reviews Neurology, vol. 6, no. 6, pp. 337-346, 2010.

[19] C. J. Earley, R. P. Allen, J. R. Connor, L. Ferrucci, and J. Troncoso, "The dopaminergic neurons of the A11 system in RLS autopsy brains appear normal," Sleep Medicine, vol. 10, no. 10, pp. 1155-1157, 2009.

[20] B. McDonagh, T. King, and R. C. Guptan, "Restless legs syndrome in patients with chronic venous disorders: an untold story," Phlebology, vol. 22, no. 4, pp. 156-163, 2007.

[21] K. E. Innes, T. K. Selfe, and P. Agarwal, "Restless legs syndrome and conditions associated with metabolic dysregulation, sympathoadrenal dysfunction, and cardiovascular disease risk: a systematic review," Sleep Medicine Reviews. vol. 38, no. 5, pp. 1-31, 2011.

[22] R. Ferri, M. Zucconi, F. Rundo, K. Spruyt, M. Manconi, and L. Ferini-Strambi, "Heart rate and spectral EEG changes accompanying periodic and non-periodic leg movements during sleep," Clinical Neurophysiology, vol. 118, no. 2, pp. 438-448, 2007.

[23] F. Siddiqui, J. Strus, X. Ming, I. A. Lee, S. Chokroverty, and A. S. Walters, "Rise of blood pressure with periodic limb movements in sleep and wakefulness," Clinical Neurophysiology, vol. 118, no. 9, pp. 1923-1930, 2007.

[24] M. H. Pennestri, J. Montplaisir, R. Colombo, G. Lavigne, and P. A. Lanfranchi, "Nocturnal blood pressure changes in patients with restless legs syndrome," Neurology, vol. 68, no. 15 , pp. 1213-1218, 2007.

[25] C. Schilling, M. Schredl, P. Strobl, and M. Deuschle, "Restless legs syndrome: evidence for nocturnal hypothalamicpituitary- adrenal system activation," Movement Disorders, vol. 25, no. 8, pp. 1047-1052, 2010.

[26] M. A. Cikrikcioglu, M. Hursitoglu, H. Erkal et al., "Oxidative stress and autonomic nervous system functions in restless legs syndrome," European Journal of Clinical Investigation, vol. 41, no. 7, pp. 734-742, 2011.

[27] L. Ferrin-Strambi, F. Poccia, A. Oldani et al., "Heart rate variability during wakefulness and sleep in restless legs," Syndrome Journal of Sleep Research, vol. 9, article 63, 2000.

[28] W. H. Oertel, C. Trenkwalder, M. Zucconi et al., "State of the art in restless legs syndrome therapy: practice recommendations for treating restless legs syndrome," Movement Disorders, vol. 22, supplement 18, pp. S466-S475, 2007.

[29] K. Spiegelhalder and M. Hornyak, "Restless legs syndrome in older adults," Clinics in Geriatric Medicine, vol. 24, no. 1, pp. 167-180, 2008.

[30] C. Trenkwalder, W. A. Hening, P. Montagna et al., "Treatment of restless legs syndrome: an evidence-based review and implications for clinical practice," Movement Disorders, vol. 23, no. 16, pp. 2267-2302, 2008.
[31] D. García-Borreguero and A. M. Williams, "Dopaminergic augmentation of restless legs syndrome," Sleep Medicine Reviews, vol. 14, no. 5, pp. 339-346, 2010.

[32] S. A. Milligan and A. L. Chesson, "Restless legs syndrome in the older adult: diagnosis and management," Drugs and Aging, vol. 19, no. 10, pp. 741-751, 2002.

[33] N. Wolkove, O. Elkholy, M. Baltzan, and M. Palayew, "Sleep and aging: 2. Management of sleep disorders in older people," CMAJ, vol. 176, no. 10, pp. 1449-1454, 2007.

[34] V. Cochen, C. Arbus, M. E. Soto et al., "Sleep disorders and their impacts on healthy, dependent, and frail older adults," Journal of Nutrition, Health and Aging, vol. 13, no. 4, pp. 322329, 2009.

[35] J. W. Winkelman and L. Johnston, "Augmentation and tolerance with long-term pramipexole treatment of restless legs syndrome (RLS)," Sleep Medicine, vol. 5, no. 1, pp. 9-14, 2004.

[36] D. Garcia-Borreguero, R. Allen, R. Kohnen et al., "Loss of response during long-term treatment of restless legs syndrome: guidelines approved by the International Restless Legs Syndrome Study Group for use in clinical trials," Sleep Medicine, vol. 11, no. 9, pp. 956-957, 2010.

[37] G. K. Sakkas, G. M. Hadjigeorgiou, C. Karatzaferi et al., "Intradialytic aerobic exercise training ameliorates symptoms of restless legs syndrome and improves functional capacity in patients on hemodialysis: a pilot study," ASAIO Journal, vol. 54, no. 2, pp. 185-190, 2008.

[38] M. M. Aukerman, D. Aukerman, M. Bayard, F. Tudiver, L. Thorp, and B. Bailey, "Exercise and restless legs syndrome: a randomized controlled trial," Journal of the American Board of Family Medicine, vol. 19, no. 5, pp. 487-493, 2006.

[39] M. Hornyak, C. Grossmann, R. Kohnen et al., "Cognitive behavioural group therapy to improve patients' strategies for coping with restless legs syndrome: a proof-of-concept trial," Journal of Neurology, Neurosurgery and Psychiatry, vol. 79, no. 7, pp. 823-825, 2008.

[40] P. M. Barnes, B. Bloom, and R. L. Nahin, "Complementary and alternative medicine use among adults and children: United States, 2007," National Health Statistics Reports, no. 12, pp. 1-23, 2009.

[41] K. Yang, "A review of yoga programs for four leading risk factors of chronic diseases," Evidence-Based Complementary and Alternative Medicine, vol. 4, no. 4, pp. 487-491, 2007.

[42] K. E. Innes and H. K. Vincent, "The influence of yoga-based programs on risk profiles in adults with type 2 diabetes mellitus: a systematic review," Evidence-Based Complementary and Alternative Medicine, vol. 4, no. 4, pp. 469-486, 2007.

[43] L. A. Gordon, E. Y. Morrison, D. A. McGrowder et al., "Effect of exercise therapy on lipid profile and oxidative stress indicators in patients with type 2 diabetes," BMC Complementary and Alternative Medicine, vol. 8, article 21, 2008.

[44] A. Ross and S. Thomas, "The health benefits of yoga and exercise: a review of comparison studies," Journal of Alternative and Complementary Medicine, vol. 16, no. 1, pp. 3-12, 2010.

[45] K. E. Innes, T. K. Selfe, and A. G. Taylor, "Menopause, the metabolic syndrome, and mind-body therapies," Menopause, vol. 15 , no. 5, pp. 1005-1013, 2008.

[46] S. B. S. Khalsa, S. M. Shorter, S. Cope, G. Wyshak, and E. Sklar, "Yoga ameliorates performance anxiety and mood disturbance in young professional musicians," Applied Psychophysiology Biofeedback, vol. 34, no. 4, pp. 279-289, 2009. 
[47] L. D. Butler, L. C. Waelde, T. A. Hastings et al., "Meditation with yoga, group therapy with hypnosis, and psychoeducation for long-term depressed mood: a randomized pilot trial," Journal of Clinical Psychology, vol. 64, no. 7, pp. 806-820, 2008.

[48] K. M. Chen, M. H. Chen, M. H. Lin, J. T. Fan, H. S. Lin, and C. H. Li, "Effects of yoga on sleep quality and depression in elders in assisted living facilities," The Journal of Nursing Research, vol. 18, no. 1, pp. 53-61, 2010.

[49] K. E. Innes, T. K. Selfe, and A. Vishnu, "Mind-body therapies for menopausal symptoms: a systematic review," Maturitas, vol. 66, no. 2, pp. 135-149, 2010.

[50] K. M. Chen, M. H. Chen, H. C. Chao, H. M. Hung, H. S. Lin, and C. H. Li, "Sleep quality, depression state, and health status of older adults after silver yoga exercises: cluster randomized trial," International Journal of Nursing Studies, vol. 46, no. 2, pp. 154-163, 2009.

[51] M. Yurtkuran, A. Alp, M. Yurtkuran, and K. Dilek, "A modified yoga-based exercise program in hemodialysis patients: a randomized controlled study," Complementary Therapies in Medicine, vol. 15, no. 3, pp. 164-171, 2007.

[52] O. P. Bhatnagar and V. Anantharaman, "The effect of Yoga training on neuromuscular excitability and muscular relaxation," Neurology India, vol. 25, no. 4, pp. 230-232, 1977.

[53] F. Tergau, S. Wischer, and W. Paulus, "Motor system excitability in patients with restless legs syndrome," Neurology, vol. 52, no. 5, pp. 1060-1063, 1999.

[54] A. Scalise, I. Pittaro-Cadore, F. Janes, R. Marinig, and G. L. Gigli, "Changes of cortical excitability after dopaminergic treatment in restless legs syndrome," Sleep Medicine, vol. 11, no. 1, pp. 75-81, 2010.

[55] K. R. Chaudhuri, "The restless legs syndrome: time to recognize a very common movement disorder," Practical Neurology, vol. 3, no. 4, pp. 204-213, 2003.

[56] D. MacMahon, S. Muzerengi, and K. R. Chaudhuri, "Treatment and identification of restless legs syndrome," Prescriber, vol. 5, pp. 56-59, 2008.

[57] K. E. Innes, T. K. Selfe, G. K. Alexander, and A. G. Taylor, "A new educational film control for use in studies of active mind-body therapies: acceptability and feasibility," Journal of Alternative and Complementary Medicine, vol. 17, no. 5, pp. 453-458, 2011.

[58] D. J. Buysse, C. F. Reynolds, T. H. Monk, S. R. Berman, and D. J. Kupfer, "The Pittsburgh Sleep Quality Index: a new instrument for psychiatric practice and research," Psychiatry Research, vol. 28, no. 2, pp. 193-213, 1989.

[59] S. Cohen, T. Kamarck, and R. Mermelstein, "A global measure of perceived stress," Journal of Health and Social Behavior, vol. 24, no. 4, pp. 385-396, 1983.

[60] B. G. Berger and R. W. Motl, "Exercise and mood: a selective review and synthesis of research employing the Profile of Mood States," Journal of Applied Sport Psychology, vol. 12, no. 1, pp. 69-92, 2000.

[61] N. V. Ramanaiah, M. Franzen, and T. Schill, "A psychometric study of the State-Trait Anxiety Inventory," Journal of Personality Assessment, vol. 47, no. 5, pp. 531-535, 1983.

[62] J. Backhaus, K. Junghanns, A. Broocks, D. Riemann, and F. Hohagen, "Test-retest reliability and validity of the Pittsburgh Sleep Quality Index in primary insomnia," Journal of Psychosomatic Research, vol. 53, no. 3, pp. 737-740, 2002.

[63] D. Picchietti and J. W. Winkelman, "Restless legs syndrome, periodic limb movements in sleep, and depression," Sleep, vol. 28, no. 7, pp. 891-898, 2005.
[64] A. Ströhle, "Physical activity, exercise, depression and anxiety disorders," Journal of Neural Transmission, vol. 116, no. 6, pp. 777-784, 2009.

[65] R. A. Washburn, E. McAuley, J. Katula, S. L. Mihalko, and R. A. Boileau, "The physical activity scale for the elderly (PASE): evidence for validity, Journal of Clinical Epidemiology, vol. 52, no. 7, pp. 643-651, 1999.

[66] H. G. Koenig, R. E. Westlund, L. K. George, D. C. Hughes, D. G. Blazer, and C. Hybels, "Abbreviating the Duke Social Support Index for use in chronically ill elderly individuals," Psychosomatics, vol. 34, no. 1, pp. 61-69, 1993.

[67] J. Cohen, Statistical Power Analysis for the Behavioral Sciences, Lawrence Erlbaum Associates, Hillsdale, NJ, USA, 2nd edition, 1988.

[68] D. L. Cohen, L. T. Bloedon, R. L. Rothman et al., "Iyengar yoga versus enhanced usual care on blood pressure in patients with prehypertension to stage $\mathrm{i}$ hypertension: a randomized controlled trial," Evidence-based Complementary and Alternative Medicine, vol. 2011, Article ID 546428, 8 pages, 2011.

[69] K. E. Innes, C. Bourguignon, and A. G. Taylor, "Risk indices associated with the insulin resistance syndrome, cardiovascular disease, and possible protection with yoga: a systematic review," Journal of the American Board of Family Practice, vol. 18, no. 6, pp. 491-519, 2005.

[70] L. G. Saptharishi, M. B. Soudarssanane, D. Thiruselvakumar et al., "Community-based randomized controlled trial of non-pharmacological interventions in prevention and control of hypertension among young adults," Indian Journal of Community Medicine, vol. 34, no. 4, pp. 329-334, 2009.

[71] M. Saletu, P. Anderer, B. Högl et al., "Acute double-blind, placebo-controlled sleep laboratory and clinical follow-up studies with a combination treatment of rr-L-dopa and sr-L-dopa in restless legs syndrome," Journal of Neural Transmission, vol. 110, no. 6, pp. 611-626, 2003.

[72] Y. Inoue, K. Kuroda, K. Hirata, N. Uchimura, T. Kagimura, and T. Shimizu, "Long-term open-label study of pramipexole in patients with primary restless legs syndrome," Journal of the Neurological Sciences, vol. 294, no. 1-2, pp. 62-66, 2010.

[73] S. Happe, G. Klösch, B. Saletu, and J. Zeitlhofer, "Treatment of idiopathic restless legs syndrome (RLS) with gabapentin," Neurology, vol. 57, no. 9, pp. 1717-1719, 2001.

[74] R. K. Bogan, J. M. Fry, M. H. Schmidt, S. W. Carson, and S. Y. Ritchie, "Ropinirole in the treatment of patients with restless legs syndrome: a US-based randomized, double-blind, placebo-controlled clinical trial," Mayo Clinic Proceedings, vol. 81, no. 1, pp. 17-27, 2006.

[75] M. Partinen, K. Hirvonen, L. Jama et al., "Efficacy and safety of pramipexole in idiopathic restless legs syndrome: a polysomnographic dose-finding study-The PRELUDE study," Sleep Medicine, vol. 7, no. 5, pp. 407-417, 2006.

[76] P. L. McCormack and M. A. A. Siddiqui, "Pramipexole: in restless legs syndrome," CNS Drugs, vol. 21, no. 5, pp. 429440, 2007.

[77] D. Garcia-Borreguero, O. Larrosa, Y. De la Llave, K. Verger, X. Masramon, and G. Hernandez, "Treatment of restless legs syndrome with gabapentin: a double-blind, cross-over study," Neurology, vol. 59, no. 10, pp. 1573-1579, 2002.

[78] F. Tison, A. Crochard, D. Léger, S. Bouée, E. Lainey, and A. El Hasnaoui, "Epidemiology of restless legs syndrome in French adults - a nationwide survey: the INSTANT Study," Neurology, vol. 65, no. 2, pp. 239-246, 2005.

[79] R. P. Allen, A. S. Walters, J. Montplaisir et al., "Restless legs syndrome prevalence and impact: REST general population 
study," Archives of Internal Medicine, vol. 165, no. 11, pp. 1286-1292, 2005.

[80] R. P. Allen, P. Stillman, and A. J. Myers, "Physician-diagnosed restless legs syndrome in a large sample of primary medical care patients in western Europe: prevalence and characteristics," Sleep Medicine, vol. 11, no. 1, pp. 31-37, 2010.

[81] K. Berger, J. Luedemann, C. Trenkwalder, U. John, and C. Kessler, "Sex and the risk of restless legs syndrome in the general population," Archives of Internal Medicine, vol. 164, no. 2, pp. 196-202, 2004.

[82] H. B. Lee, W. A. Hening, R. P. Allen, C. J. Earley, W. W. Eaton, and C. G. Lyketsos, "Race and restless legs syndrome symptoms in an adult community sample in east Baltimore," Sleep Medicine, vol. 7, no. 8, pp. 642-645, 2006.

[83] J. W. Winkelman, L. Finn, and T. Young, "Prevalence and correlates of restless legs syndrome symptoms in the Wisconsin Sleep Cohort," Sleep Medicine, vol. 7, no. 7, pp. 545-552, 2006.

[84] H. Hachul, L. R. A. Bittencourt, M. L. Andersen, M. A. Haidar, E. C. Baracat, and S. Tufik, "Effects of hormone therapy with estrogen and/or progesterone on sleep pattern in postmenopausal women," International Journal of Gynecology and Obstetrics, vol. 103, no. 3, pp. 207-212, 2008.

[85] P. Polo-Kantola and R. Erkkola, "Sleep and the menopause," Journal of the British Menopause Society, vol. 10, no. 4, pp. 145-150, 2004.

[86] G. Saletu-Zyhlarz, P. Anderer, G. Gruber et al., "Insomnia related to postmenopausal syndrome and hormone replacement therapy: sleep laboratory studies on baseline differences between patients and controls and double-blind, placebocontrolled investigations on the effects of a novel estrogenprogestogen combination (Climodien, Lafamme) versus estrogen alone," Journal of Sleep Research, vol. 12, no. 3, pp. 239-254, 2003.

[87] G. Weitz, M. Elam, J. Born, H. L. Fehm, and C. Dodt, "Postmenopausal estrogen administration suppresses muscle sympathetic nerve activity," Journal of Clinical Endocrinology and Metabolism, vol. 86, no. 1, pp. 344-348, 2001.

[88] A. J. Rothdach, C. Trenkwalder, J. Haberstock, U. Keil, and K. Berger, "Prevalence and risk factors of RLS in an elderly population: the MEMO study," Neurology, vol. 54, no. 5, pp. 1064-1068, 2000.

[89] J. Wesström, S. Nilsson, I. Sundström-Poromaa, and J. Ulfberg, "Restless legs syndrome among women: prevalence, co-morbidity and possible relationship to menopause," Climacteric, vol. 11, no. 5, pp. 422-428, 2008.

[90] I. Ghorayeb, B. Bioulac, C. Scribans, and F. Tison, "Perceived severity of restless legs syndrome across the female life cycle," Sleep Medicine, vol. 9, no. 7, pp. 799-802, 2008.

[91] B. S. McEwen, "Physiology and neurobiology of stress and adaptation: central role of the brain," Physiological Reviews, vol. 87, no. 3, pp. 873-904, 2007.

[92] B. S. McEwen, "Sleep deprivation as a neurobiologic and physiologic stressor: allostasis and allostatic load," Metabolism, vol. 55, supplement 1, pp. S20-S23, 2006.

[93] R. P. Brown and P. L. Gerbarg, "Sudarshan Kriya yogic breathing in the treatment of stress, anxiety, and depression: part I-neurophysiologic model," Journal of Alternative and Complementary Medicine, vol. 11, no. 1, pp. 189-201, 2005.

[94] K. E. Innes, H. K. Vincent, and A. G. Taylor, "Chronic stress and insulin resistance-related indices of cardiovascular disease risk, part I: neurophysiological responses and pathological sequelae," Alternative Therapies in Health and Medicine, vol. 13, no. 4, pp. 46-52, 2007.

[95] K. E. Innes, H. K. Vincent, and A. G. Taylor, "Chronic stress and insulin resistance-related indices of cardiovascular disease risk, part 2: a potential role for mind-body therapies," Alternative Therapies in Health and Medicine, vol. 13, no. 5, pp. 44-51, 2007.

[96] D. L. Cohen, N. Wintering, V. Tolles et al., "Cerebral blood flow effects of yoga training: preliminary evaluation of 4 cases," Journal of Alternative and Complementary Medicine, vol. 15, no. 1, pp. 9-14, 2009.

[97] C. C. Streeter, J. E. Jensen, R. M. Perlmutter et al., "Yoga Asana sessions increase brain GABA levels: a pilot study," Journal of Alternative and Complementary Medicine, vol. 13, no. 4, pp. 419-426, 2007.

[98] T. W. Kjaer, C. Bertelsen, P. Piccini, D. Brooks, J. Alving, and H. C. Lou, "Increased dopamine tone during meditationinduced change of consciousness," Cognitive Brain Research, vol. 13, no. 2, pp. 255-259, 2002.

[99] S. Leknes and I. Tracey, "A common neurobiology for pain and pleasure," Nature Reviews Neuroscience, vol. 9, no. 4, pp. 314-320, 2008.

[100] E. Mignot, S. Taheri, and S. Nishino, "Sleeping with the hypothalamus: emerging therapeutic targets for sleep disorders," Nature Neuroscience, vol. 5, pp. 1071-1075, 2002.

[101] I. K. Goerendt, C. Messa, A. D. Lawrence, P. M. Grasby, P. Piccini, and D. J. Brooks, "Dopamine release during sequential finger movements in health and Parkinson's disease: a PET study," Brain, vol. 126, no. 2, pp. 312-325, 2003.

[102] R. Scranton and A. Cincotta, "Bromocriptine unique formulation of a dopamine agonist for the treatment of type 2 diabetes," Expert Opinion on Pharmacotherapy, vol. 11, no. 2, pp. 269-279, 2010.

[103] J. M. Belavic, "A new treatment option for type 2 diabetes," The Nurse Practitioner, vol. 35, no. 1, pp. 51-52, 2010.

[104] K. G. Lloyd and P. Worms, "Neuropharmacological actions of GABA agonists: predictability for their clinical usefulness," Advances in Biochemical Psychopharmacology, vol. 29, pp. 5967, 1981.

[105] I. S. Shiah and L. N. Yatham, "GABA function in mood disorders: an update and critical review," Life Sciences, vol. 63, no. 15, pp. 1289-1303, 1998.

[106] S. J. Enna and K. E. McCarson, "The role of GABA in the mediation and perception of pain," Advances in Pharmacology, vol. 54, pp. 1-27, 2006.

[107] G. Chouinard, "The search for new off-label indications for antidepressant, antianxiety, antipsychotic and anticonvulsant drugs," Journal of Psychiatry and Neuroscience, vol. 31, no. 3, pp. 168-176, 2006.

[108] L. M. Arnold, D. L. Goldenberg, S. B. Stanford et al., "Gabapentin in the treatment of fibromyalgia: a randomized, double-blind, placebo-controlled, multicenter trial," Arthritis and Rheumatism, vol. 56, no. 4, pp. 1336-1344, 2007.

[109] J. K. Baillie and I. Power, "The mechanism of action of gabapentin in neuropathic pain," Current Opinion in Investigational Drugs, vol. 7, no. 1, pp. 33-39, 2006.

[110] S. Fulda and T. C. Wetter, "Where dopamine meets opioids: a meta-analysis of the placebo effect in restless legs syndrome treatment studies," Brain, vol. 131, part 4, pp. 902-917, 2008.

[111] H. Benes, A. S. Walters, R. P. Allen, W. A. Hening, and R. Kohnen, "Definition of restless legs syndrome, how to diagnose it, and how to differentiate it from RLS mimics," 
Movement Disorders, vol. 22, supplement 18, pp. S401-S408, 2007.

[112] F. Brindani, F. Vitetta, and F. Gemignani, "Restless legs syndrome: differential diagnosis and management with pramipexole," Clinical Interventions in Aging, vol. 4, pp. 305313, 2009.

[113] R. A. Hansen, L. Song, C. G. Moore et al., "Effect of ropinirole on sleep outcomes in patients with restless legs syndrome: meta-Analysis of pooled individual patient data from randomized controlled trials," Pharmacotherapy, vol. 29, no. 3, pp. 255-262, 2009.

[114] C. F. Conti, M. M. De Oliveira, R. B. Andriolo et al., "Levodopa for idiopathic restless legs syndrome: evidence-based review," Movement Disorders, vol. 22, no. 13, pp. 1943-1951, 2007.

[115] C. F. Conti, M. M. De Oliveira, J. S. Valbuza, L. B. F. Prado, L. B. C. De Carvalho, and G. F. D. Prado, "Anticonvulsants to treat idiopathic restless legs syndrome: systematic review," Arquivos de Neuro-Psiquiatria, vol. 66, no. 2, pp. 431-435, 2008.

[116] C. A. Kushida, "Ropinirole for the treatment of restless legs syndrome," Neuropsychiatric Disease and Treatment, vol. 2, no. 4, pp. 407-419, 2006.

[117] J. W. Winkelman, S. Redline, C. M. Baldwin, H. E. Resnick, A. B. Newman, and D. J. Gottlieb, "Polysomnographic and health-related quality of life correlates of restless legs syndrome in the sleep heart health study," Sleep, vol. 32, no. 6, pp. 772-778, 2009.

[118] M. Hornyak, M. Kopasz, M. Berger, D. Riemann, and U. Voderholzer, "Impact of sleep-related complaints on depressive symptoms in patients with restless legs syndrome," Journal of Clinical Psychiatry, vol. 66, no. 9, pp. 1139-1145, 2005.

[119] W. Hening, A. S. Walters, R. P. Allen, J. Montplaisir, A. Myers, and L. Ferini-Strambi, "Impact, diagnosis and treatment of restless legs syndrome (RLS) in a primary care population: the REST (RLS epidemiology, symptoms, and treatment) primary care study," Sleep Medicine, vol. 5, no. 3, pp. 237246, 2004. 


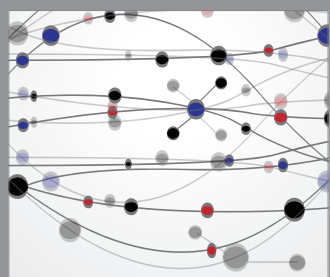

The Scientific World Journal
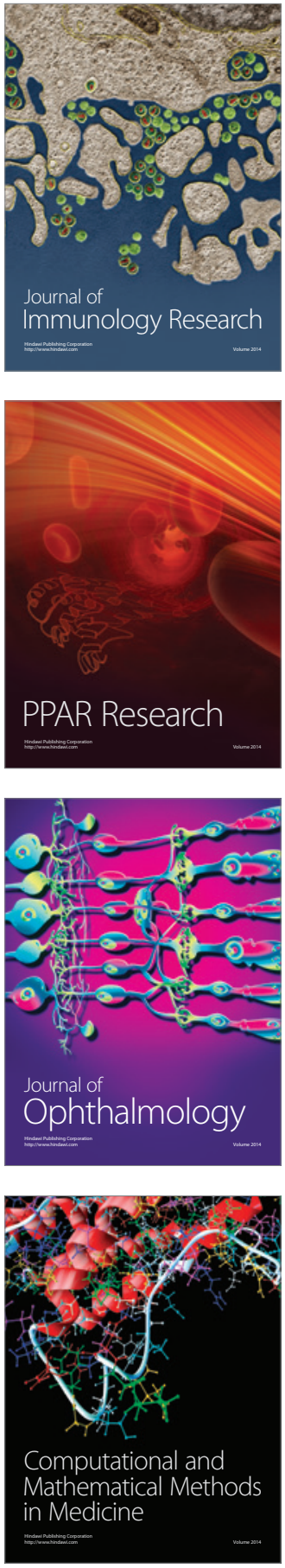

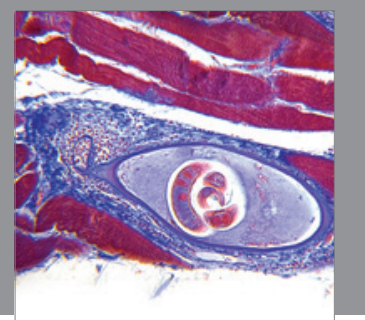

Gastroenterology

Research and Practice
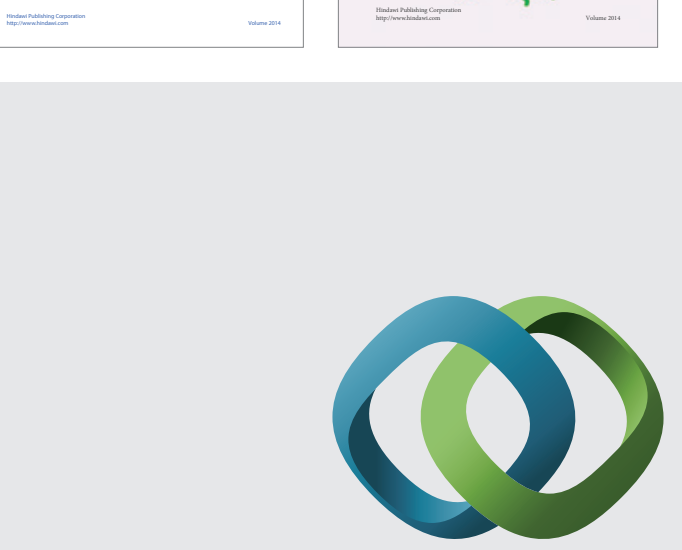

\section{Hindawi}

Submit your manuscripts at

http://www.hindawi.com
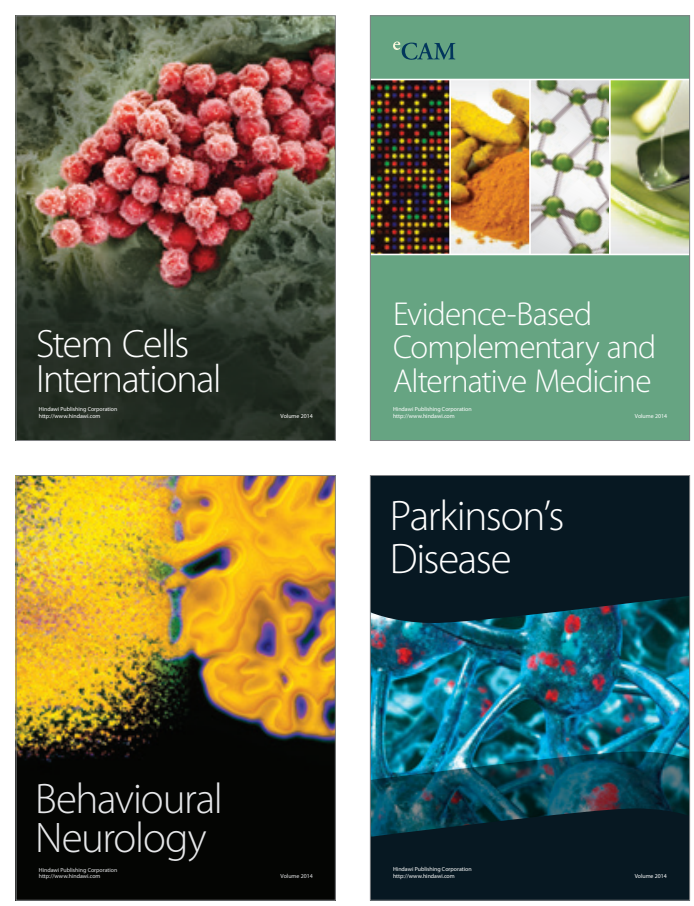

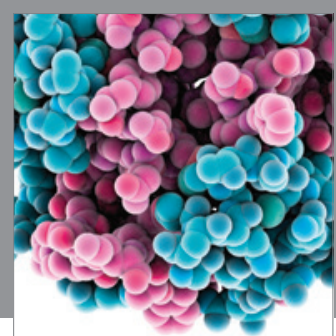

Journal of
Diabetes Research

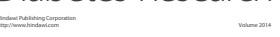

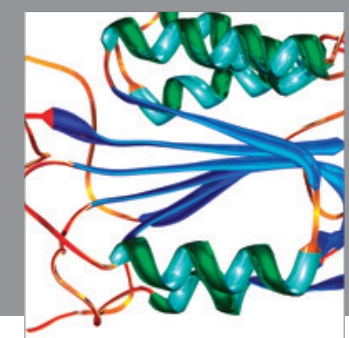

Disease Markers
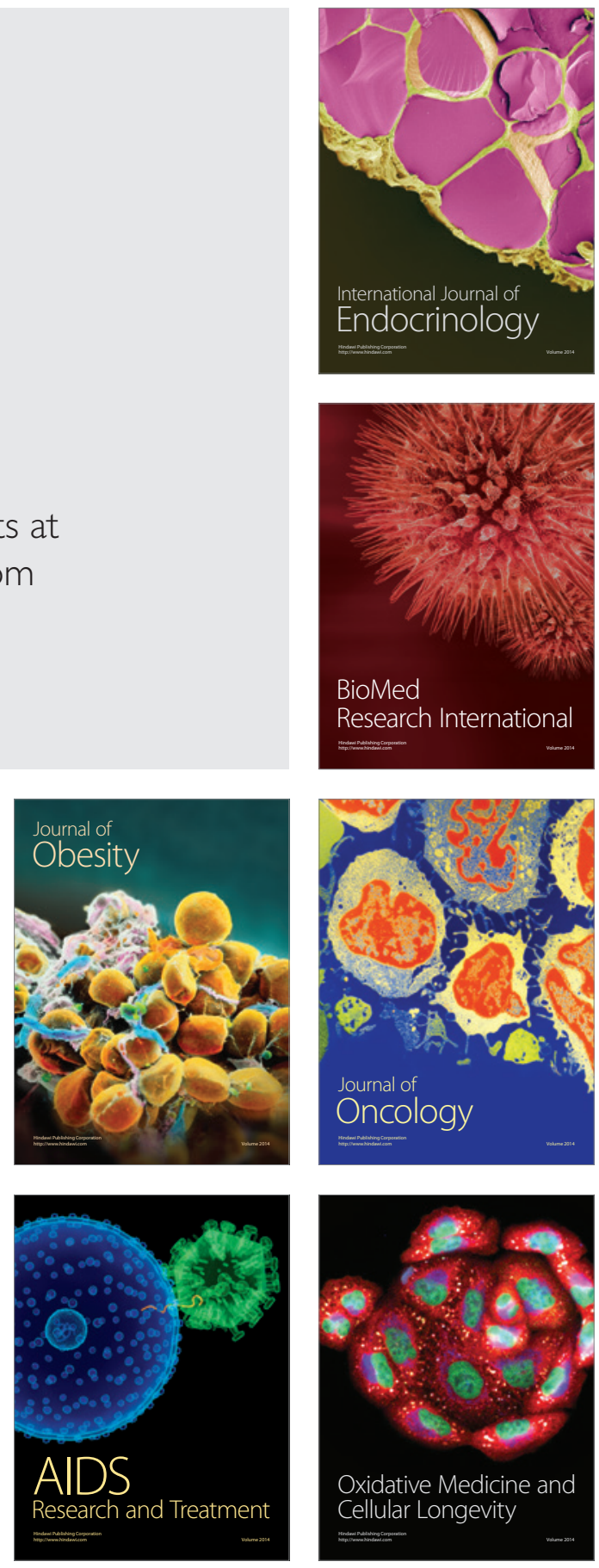